\title{
Research Article \\ Optimality Conditions and Duality in Nonsmooth Multiobjective Programs
}

\author{
Do Sang Kim and Hyo Jung Lee \\ Division of Mathematical Sciences, Pukyong National University, Busan 608-737, South Korea \\ Correspondence should be addressed to Do Sang Kim, dskim@pknu.ac.kr
}

Received 29 October 2009; Accepted 14 March 2010

Academic Editor: Jong Kyu Kim

Copyright (C) 2010 D. S. Kim and H. J. Lee. This is an open access article distributed under the Creative Commons Attribution License, which permits unrestricted use, distribution, and reproduction in any medium, provided the original work is properly cited.

We study nonsmooth multiobjective programming problems involving locally Lipschitz functions and support functions. Two types of Karush-Kuhn-Tucker optimality conditions with support functions are introduced. Sufficient optimality conditions are presented by using generalized convexity and certain regularity conditions. We formulate Wolfe-type dual and Mond-Weirtype dual problems for our nonsmooth multiobjective problems and establish duality theorems for (weak) Pareto-optimal solutions under generalized convexity assumptions and regularity conditions.

\section{Introduction}

Multiobjective programming problems arise when more than one objective function is to be optimized over a given feasible region. Pareto optimum is the optimality concept that appears to be the natural extension of the optimization of a single objective to the consideration of multiple objectives.

In 1961, Wolfe [1] obtained a duality theorem for differentiable convex programming. Afterwards, a number of different duals distinct from the Wolfe dual are proposed for the nonlinear programs by Mond and Weir [2]. Duality relations for multiobjective programming problems with generalized convexity conditions were given by several authors [3-10]. Majumdar [11] gave sufficient optimality conditions for differentiable multiobjective programming which modified those given in Singh [12] under the assumption of convexity, pseudoconvexity, and quasiconvexity of the functions involved at the Pareto-optimal solution. Subsequently, Kim et al. [13] gave a counterexample showing that some theorems of Majumdar [11] are incorrect and establish sufficient optimality theorems for (weak) Paretooptimal solutions by using modified conditions. Later on, Kim and Schaible [6] introduced 
nonsmooth multiobjective programming problems involving locally Lipschitz functions for inequality and equality constraints. They extended sufficient optimality conditions in Kim et al. [13] to the nonsmooth case and established duality theorems for nonsmooth multiobjective programming problems involving locally Lipschitz functions.

In this paper, we apply the results in Kim and Schaible [6] for this problem to nonsmooth multiobjective programming problem involving support functions. We introduce nonsmooth multiobjective programming problems involving locally Lipschitz functions and support functions for inequality and equality constraints. Two kinds of sufficient optimality conditions under various convexity assumptions and certain regularity conditions are presented. We propose a Wolfe-type dual and a Mond-Weir-type dual for the primal problem and establish duality results between the primal problem and its dual problems under generalized convexity and regularity conditions.

\section{Notation and Definitions}

Let $\mathbb{R}^{n}$ be the $n$-dimensional Euclidean space and $\mathbb{R}_{+}^{n}$ its nonnegative orthant.

We consider the following nonsmooth multiobjective programming problem involving locally Lipschitz functions:

$$
\begin{array}{ll}
\text { minimize } & f(x)+s(x \mid C)=\left(f_{1}(x)+s\left(x C_{1}\right), \ldots, f_{m}(x)+s\left(x \mid C_{m}\right)\right) \\
\text { subject to } & g_{j}(x) \leqq 0, \quad j \in P, \quad h_{k}(x)=0, \quad k \in Q, \quad x \in \mathbb{R}^{n}
\end{array}
$$

where $f_{i}: \mathbb{R}^{n} \rightarrow \mathbb{R}, i \in M=\{1,2, \ldots, m\}, g_{j}: \mathbb{R}^{n} \rightarrow \mathbb{R}, j \in P=\{1,2, \ldots, p\}$, and $h_{k}: \mathbb{R}^{n} \rightarrow \mathbb{R}$, $k \in Q=\{1,2, \ldots, q\}$, are locally Lipschitz functions. Here, $C_{i}, i \in M$, is compact convex sets in $\mathbb{R}^{n}$. We accept the formal writing $C=\left(C_{1}, C_{2}, \ldots, C_{m}\right)^{t}$ with the convention that $s(x \mid C)=$ $\left(s\left(x \mid C_{1}\right), \ldots, s\left(x \mid C_{m}\right)\right)$, where $s\left(x \mid C_{i}\right)$ is the support function of $C_{i}$ (see Definition 2.2).

Throughout the article the following notation for order relations in $\mathbb{R}^{n}$ will be used:

$$
\begin{gathered}
x \leqq u \Longleftrightarrow u-x \in \mathbb{R}_{+}^{n}, \\
x \leq u \Longleftrightarrow u-x \in \mathbb{R}_{+}^{n} \backslash\{0\}, \\
x<u \Longleftrightarrow u-x \in \operatorname{int} \mathbb{R}_{+}^{n},
\end{gathered}
$$

$x \notin u$ is the negation of $x \leq u$,

$x \nless u$ is the negation of $x<u$.

Definition 2.1. (i) A real-valued function $F: \mathbb{R}^{n} \rightarrow \mathbb{R}$ is said to be locally Lipschitz if for each $z \in \mathbb{R}^{n}$, there exist a positive constant $K$ and a neighborhood $N$ of $z$ such that, for every $x, y \in N$,

$$
|F(x)-F(y)| \leqq K\|x-y\|,
$$

where $\|\cdot\|$ denotes any norm in $\mathbb{R}^{n}$. 
(ii) The Clarke generalized directional derivative [14] of a locally Lipschitz function $F$ at $x$ in the direction $d \in \mathbb{R}^{n}$, denoted by $F^{0}(x ; d)$, is defined as follows:

$$
F^{0}(x ; d)=\limsup _{y \rightarrow x, t \rightarrow 0+} t^{-1}(F(y+t d)-F(y))
$$

where $y$ is a vector in $\mathbb{R}^{n}$.

(iii) The Clarke generalized subgradient [14] of $F$ at $x$ is denoted by

$$
\partial F(x)=\left\{\xi \in \mathbb{R}^{n}: F^{0}(x ; d) \geqq \xi^{t} d, \forall d \in \mathbb{R}^{n}\right\} .
$$

(iv) $F$ is said to be regular at $x$ if for all $d \in \mathbb{R}^{n}$ the one-sided directional derivative $F^{\prime}(x ; d)$ exists and $F^{\prime}(x ; d)=F^{0}(x ; d)$.

Definition 2.2 (see [10]). Let $C$ be a compact convex set in $\mathbb{R}^{n}$. The support function $s(x \mid C)$ of $C$ is defined by

$$
s(x \mid C):=\max \left\{x^{t} y: y \in C\right\} .
$$

The support function $s(x \mid C)$, being convex and everywhere finite, has a subdifferential, that is, for every $x \in \mathbb{R}^{n}$ there exists $z$ such that

$$
s(y \mid C) \geq s(x \mid C)+z^{t}(y-x), \quad \forall y \in C .
$$

Equivalently,

$$
z^{t} x=s(x \mid C)
$$

The subdifferential of $s(x \mid C)$ is given by

$$
\partial s(x \mid C):=\left\{z \in C: z^{t} x=s(x \mid C)\right\}
$$

For any set $S \subset \mathbb{R}^{n}$, the normal cone to $S$ at a point $x \in S$ is defined by

$$
N_{S}(x):=\left\{y \in \mathbb{R}^{n}: y^{t}(z-x) \leq 0, \forall z \in S\right\}
$$

It is readily verified that for a compact convex set $C, y$ is in $N_{C}(x)$ if and only if $s(y \mid C)=x^{t} y$, or equivalently, $x$ is in the subdifferential of $s$ at $y$.

In the notation of the problem (MP), we recall the definitions of convexity, affine, pseudoconvexity, and quasiconvexity for locally Lipschitz functions.

Definition 2.3. (i) $f=\left(f_{1}, f_{2}, \ldots, f_{m}\right)$ is convex (strictly convex) at $x^{0} \in X$ if for each $x \in X$ and any $\xi_{i} \in \partial f_{i}\left(x^{0}\right), f_{i}(x)-f_{i}\left(x^{0}\right) \geqq(>) \xi_{i}^{t}\left(x-x^{0}\right)$, for all $i \in M$. 
(ii) $g_{A}$ is convex at $x^{0} \in X$ if for each $x \in X$ and any $\zeta_{j} \in \partial g_{j}\left(x^{0}\right), g_{j}(x)-g_{j}\left(x^{0}\right) \geqq$ $\zeta_{j}^{t}\left(x-x^{0}\right)$, where $j \in A$, and $g_{A}$ denotes the active constraints at $x^{0}$.

(iii) $h=\left(h_{1}, h_{2}, \ldots, h_{k}\right)$ is convex at $x^{0} \in X$ if for each $x \in X$ and any $\alpha_{k} \in \partial h_{k}\left(x^{0}\right)$, $h_{k}(x)-h_{k}\left(x^{0}\right) \geqq \alpha_{k}^{t}\left(x-x^{0}\right)$, for all $k \in Q$.

(iv) $h$ is affine at $x^{0} \in X$ if for each $x \in X$ and any $\alpha_{k} \in \partial h_{k}\left(x^{0}\right), h_{k}(x)-h_{k}\left(x^{0}\right)=$ $\alpha_{k}^{t}\left(x-x^{0}\right)$, for all $k \in Q$.

(v) $f$ is pseudoconvex at $x^{0} \in X$ if for each $x \in X$ and any $\xi_{i} \in \partial f_{i}\left(x^{0}\right), \xi_{i}^{t}\left(x-x^{0}\right) \geqq 0$ implies $f_{i}(x) \geqq f_{i}\left(x^{0}\right)$, for all $i \in M$.

(vi) $f$ is strictly pseudoconvex at $x^{0} \in X$ if for each $x \in X$ with $x \neq x^{0}$ and any $\xi_{i} \in$ $\partial f_{i}\left(x^{0}\right), \xi_{i}^{t}\left(x-x^{0}\right) \geqq 0$ implies $f_{i}(x)>f_{i}\left(x^{0}\right)$, for all $i \in M$.

(vii) $f$ is quasiconvex at $x^{0} \in X$ if for each $x \in X$ with $x \neq x^{0}$ and any $\xi_{i} \in \partial f_{i}\left(x^{0}\right)$, $f_{i}(x) \leqq f_{i}\left(x^{0}\right)$ implies $\xi_{i}^{t}\left(x-x^{0}\right) \leqq 0$, for all $i \in M$.

Finally, we recall the definition of Pareto-optimal (efficient, nondominated) and weak Pareto-optimal solutions of (MP).

Definition 2.4. (i) A point $x^{0} \in X$ is said to be a Pareto-optimal solution of (MP) if there exists no other $x \in X$ with $f(x) \leq f\left(x^{0}\right)$.

(ii) A point $x^{0} \in X$ is said to be a weak Pareto-optimal solution of (MP) if there exists no other $x \in X$ with $f(x)<f\left(x^{0}\right)$.

\section{Sufficient Optimality Conditions}

In this section, we introduce the following two types of (KKT) conditions which differ only in the nonnegativity of the multipliers for the equality constraint and neither of which includes a complementary slackness condition, common in necessary optimality conditions [14].

$$
\begin{aligned}
& \exists u^{0} \geq 0, v^{0} \geqq 0, w^{0} \geqq 0\left(u^{0} \in \mathbb{R}^{m}, v^{0} \in \mathbb{R}^{p}, w^{0} \in \mathbb{R}^{q}\right) \text { such that } \\
& 0 \in \sum_{i \in M} u_{i}^{0}\left(\partial f_{i}\left(x^{0}\right)+z_{i}\right)+\sum_{j \in A} v_{j}^{0} \partial g_{j}\left(x^{0}\right)+\sum_{k \in Q} w_{k}^{0} \partial h_{k}\left(x^{0}\right), \\
& g\left(x^{0}\right) \leqq 0, \quad h\left(x^{0}\right)=0, \quad z_{i}^{t} x_{i}^{0}=s\left(x^{0} \mid C_{i}\right), \quad i \in M, \\
& \text { where } A=\left\{j \in P: g_{j}\left(x^{0}\right)=0\right\} . \\
& \exists u^{0} \geq 0, v^{0} \geqq 0, w^{0}\left(u^{0} \in \mathbb{R}^{m}, v^{0} \in \mathbb{R}^{p}, w^{0} \in \mathbb{R}^{q}\right) \text { such that } \\
& 0 \in \sum_{i \in M} u_{i}^{0}\left(\partial f_{i}\left(x^{0}\right)+z_{i}\right)+\sum_{j \in A} v_{j}^{0} \partial g_{j}\left(x^{0}\right)+\sum_{k \in Q} w_{k}^{0} \partial h_{k}\left(x^{0}\right), \\
& g\left(x^{0}\right) \leqq 0, \quad h\left(x^{0}\right)=0, \quad z_{i}^{t} x_{i}^{0}=s\left(x^{0} \mid C_{i}\right), \quad i \in M, \\
& \text { where } A=\left\{j \in P: g_{j}\left(x^{0}\right)=0\right\} .
\end{aligned}
$$


In Theorems 3.1 and 3.2 and Corollaries 3.3 and 3.4 below we present new versions including support functions of the results by Kim and Schaible in [6] for smooth problems (MP) involving (KKT).

Theorem 3.1. Let $\left(x^{0}, u^{0}, v^{0}, w^{0}\right)$ satisfy (KKT). If $f(\cdot)+z^{t}(\cdot)$ is pseudoconvex at $x^{0}, g_{A}$ and $h$ are quasiconvex at $x^{0}$, and $f$ is regular at $x^{0}$, then $x^{0}$ is a weak Pareto-optimal solution of (MP).

Proof. Let $\left(x^{0}, u^{0}, v^{0}, w^{0}\right)$ satisfy (KKT). Then $g\left(x^{0}\right) \leqq 0, h\left(x^{0}\right)=0$,

$$
0 \in \sum_{i \in M} u_{i}^{0}\left(\partial f_{i}\left(x^{0}\right)+z_{i}\right)+\sum_{j \in A} v_{j}^{0} \partial g_{j}\left(x^{0}\right)+\sum_{k \in Q} w_{k}^{0} \partial h_{k}\left(x^{0}\right)
$$

From (3.1), there exist $\xi_{i} \in \partial f_{i}\left(x^{0}\right), \zeta_{j} \in \partial g_{j}\left(x^{0}\right)$, and $\alpha_{k} \in \partial h_{k}\left(x^{0}\right)$ such that

$$
\sum_{i \in M} u_{i}^{0}\left(\xi_{i}+z_{i}\right)+\sum_{j \in A} v_{j}^{0} \zeta_{j}+\sum_{k \in Q} w_{k}^{0} \alpha_{k}=0
$$

Suppose that $x^{0} \in X$ is not a weak Pareto-optimal solution of (MP). Then there exists $\bar{x} \in X$ such that $f(\bar{x})+s(\bar{x} \mid C)<f\left(x^{0}\right)+s\left(x^{0} \mid C\right)$ that implies $f(\bar{x})+z^{t} \bar{x}<f\left(x^{0}\right)+z^{t} x^{0}$ because of $z^{t} x \leqq s(x \mid C)$ and the assumption $z^{t} x^{0}=s\left(x^{0} \mid C\right)$ which means that this function $s(x \mid C)$ is subdifferentiable and regular at $x^{0}$. By pseudoconvexity of $f(\cdot)+z^{t}(\cdot)$ at $x^{0}$, we have

$$
\left(\xi_{i}+z_{i}\right)^{t}\left(\bar{x}-x^{0}\right)<0 \text { for any } \xi_{i} \in \partial f_{i}\left(x^{0}\right), i \in M
$$

Since $g_{j}(\bar{x}) \leqq 0=g_{j}\left(x^{0}\right), j \in A$, we obtain the following inequality with the help of quasiconvexity of $g_{A}$ at $x^{0}$ :

$$
\zeta_{j}^{t}\left(\bar{x}-x^{0}\right) \leqq 0 \quad \text { for any } \zeta_{j} \in \partial g_{j}\left(x^{0}\right), j \in A
$$

Also, since $h_{k}(\bar{x})=0=h_{k}\left(x^{0}\right), k \in Q$, it follows from quasiconvexity of $h$ at $x^{0}$ that

$$
\alpha_{k}^{t}\left(\bar{x}-x^{0}\right) \leqq 0 \quad \text { for any } \alpha_{k} \in \partial h_{k}\left(x^{0}\right), k \in Q
$$

From (3.3)-(3.5), we obtain

$$
\left[\sum_{i \in M} u_{i}^{0}\left(\xi_{i}+z_{i}\right)+\sum_{j \in A} v_{j}^{0} \zeta_{j}+\sum_{k \in Q} w_{k}^{0} \alpha_{k}\right]^{t}\left(\bar{x}-x^{0}\right)<0
$$

which contradicts (3.2). Hence $x^{0}$ is a weak Pareto-optimal solution of (MP).

Theorem 3.2. Let $\left(x^{0}, u^{0}, v^{0}, w^{0}\right)$ satisfy (KKT). If $f(\cdot)+z^{t}(\cdot)$ is strictly pseudoconvex at $x^{0}, g_{A}$ and $h$ are quasiconvex at $x^{0}$, and $f$ is regular at $x^{0}$, then $x^{0}$ is a Pareto-optimal solution of (MP). 
Corollary 3.3. Let $\left(x^{0}, u^{0}, v^{0}, w^{0}\right)$ satisfy (KKT). If $f(\cdot)+z^{t}(\cdot), g_{A}$ and hare convex at $x^{0}$, and $f$ is regular at $x^{0}$, then $x^{0}$ is a weak Pareto-optimal solution of (MP).

Corollary 3.4. Let $\left(x^{0}, u^{0}, v^{0}, w^{0}\right)$ satisfy (KKT). If $f(\cdot)+z^{t}(\cdot)$ is strictly convex at $x^{0}, g_{A}$ and $h$ are convex at $x^{0}$, and $f$ is regular at $x^{0}$, then $x^{0}$ is a Pareto-optimal solution of (MP).

Theorem 3.5. Let $\left(x^{0}, u^{0}, v^{0}, w^{0}\right)$ satisfy $\left(\mathrm{KKT}^{\prime}\right)$. If $f(\cdot)+z^{t}(\cdot)$ is quasiconvex at $x^{0},\left(v^{0}\right)^{t} g_{A}+$ $\left(w^{0}\right)^{t} h$ is strictly pseudoconvex at $x^{0}$, and $f, g_{A}$, and $h$ are regular at $x^{0}$, then $x^{0}$ is a Pareto-optimal solution of (MP).

Proof. Suppose that $x^{0} \in X$ is not a Pareto-optimal solution of (MP). Then there exists $\bar{x} \in X$ such that $f(\bar{x})+s(\bar{x} \mid C) \leq f\left(x^{0}\right)+s\left(x^{0} \mid C\right)$, that implies $f(\bar{x})+z^{t} \bar{x} \leq f\left(x^{0}\right)+z^{t} x^{0}$ because of $z^{t} x \leqq s(x \mid C)$ and the assumption $z^{t} x^{0}=s\left(x^{0} \mid C\right)$. By quasiconvexity of $f(\cdot)+z^{t}(\cdot)$ at $x^{0}$, we have

$$
\left(\xi_{i}+z_{i}\right)^{t}\left(\bar{x}-x^{0}\right) \leqq 0 \quad \text { for any } \xi_{i} \in \partial f_{i}\left(x^{0}\right), i \in M
$$

Since $\left(x^{0}, u^{0}, v^{0}, w^{0}\right)$ satisfy $\left(\mathrm{KKT}^{\prime}\right)$, we obtain $\left[\sum_{j \in A} v_{j}^{0} \zeta_{j}+\sum_{k \in Q} w_{k}^{0} \alpha_{k}\right]^{t}\left(\bar{x}-x^{0}\right) \geqq 0$ for some $\zeta_{j} \in \partial g_{j}\left(x^{0}\right), j \in A$, and $\alpha_{k} \in \partial h_{k}\left(x^{0}\right), k \in Q$. By regularity of $g_{A}$ and $h$ at $x^{0}$, there exists $\beta \in \partial\left(v^{0}\right)^{t} g_{A}+\left(w^{0}\right)^{t} h$ such that $\beta^{t}\left(\bar{x}-x^{0}\right) \geqq 0$. With the help of a strict pseudoconvexity of $\left(v^{0}\right)^{t} g_{A}+\left(w^{0}\right)^{t} h$, we have

$$
\left(v^{0}\right)^{t} g_{A}(\bar{x})+\left(w^{0}\right)^{t} h(\bar{x})>\left(v^{0}\right)^{t} g_{A}\left(x^{0}\right)+\left(w^{0}\right)^{t} h\left(x^{0}\right) .
$$

Since $\bar{x} \in X$, we obtain

$$
\left(v^{0}\right)^{t} g_{A}(\bar{x})+\left(w^{0}\right)^{t} h(\bar{x}) \leqq 0
$$

Since $g_{A}\left(x^{0}\right)=h\left(x^{0}\right)=0$, we obtain

$$
\left(v^{0}\right)^{t} g_{A}\left(x^{0}\right)+\left(w^{0}\right)^{t} h\left(x^{0}\right)=0
$$

Substituting (3.9) and (3.10) for (3.8), we arrive at a contradiction. Hence $x^{0}$ is a Paretooptimal solution of (MP).

Now we present a result for nonsmooth problems (MP) which in the smooth case is similar to Singh's earlier result in [12] under generalized convexity.

Theorem 3.6. Let $\left(x^{0}, u^{0}, v^{0}, w^{0}\right)$ satisfy $\left(\mathrm{KKT}^{\prime}\right)$. If $\left(u^{0}\right)^{t}\left(f(\cdot)+z^{t}(\cdot)\right)+\left(v^{0}\right)^{t} g_{A}+\left(w^{0}\right)^{t} h$ is pseudoconvex at $x^{0}$, and $f, g_{A}$, and $h$ are regular at $x^{0}$, then $x^{0}$ is a weak Pareto-optimal solution of (MP).

Proof. Suppose that $x^{0} \in X$ is not a weak Pareto-optimal solution of (MP). Then there exists $\bar{x} \in X$ such that $f(\bar{x})+s(\bar{x} \mid C)<f\left(x^{0}\right)+s\left(x^{0} \mid C\right)$, that implies $f(\bar{x})+z^{t} \bar{x}<f\left(x^{0}\right)+z^{t} x^{0}$ because of $z^{t} x \leqq s(x \mid C)$ and the assumption $z^{t} x^{0}=s\left(x^{0} \mid C\right)$. Since $\bar{x} \in X$, we have 
$g_{A}(\bar{x}) \leqq 0=g_{A}\left(x^{0}\right)$ and $h(\bar{x})=0=h\left(x^{0}\right)$. Therefore, $f(\bar{x})+s(\bar{x} \mid C)-f\left(x^{0}\right)+s\left(x^{0} \mid C\right)<0$, $g_{A}(\bar{x})-g_{A}\left(x^{0}\right) \leqq 0$, and $h(\bar{x})-h\left(x^{0}\right)=0$. Hence $\left(u^{0}\right)^{t}\left(f(\bar{x})+z^{t}(\bar{x})\right)+\left(v^{0}\right)^{t} g_{A}(\bar{x})+\left(w^{0}\right)^{t} h(\bar{x})<$ $\left(u^{0}\right)^{t}\left(f\left(x^{0}\right)+z^{t}\left(x^{0}\right)\right)+\left(v^{0}\right)^{t} g_{A}\left(x^{0}\right)+\left(w^{0}\right)^{t} h\left(x^{0}\right)$. Since $f, g_{A}$, and $h$ are regular at $x^{0}$, we obtain

$$
\begin{aligned}
& \partial\left(\sum_{i \in M} u_{i}^{0}\left(f_{i}\left(x^{0}\right)+s\left(x^{0} \mid C_{i}\right)\right)+\sum_{j \in A} v_{j}^{0} g_{j}\left(x^{0}\right)+\sum_{k \in Q} w_{k}^{0} h_{k}\left(x^{0}\right)\right) \\
& =\sum_{i \in M} u_{i}^{0}\left(\partial f_{i}\left(x^{0}\right)+\partial s\left(x^{0} \mid C_{i}\right)\right)+\sum_{j \in A} v_{j}^{0} \partial g_{j}\left(x^{0}\right)+\sum_{k \in Q} w_{k}^{0} \partial h_{k}\left(x^{0}\right) .
\end{aligned}
$$

By pseudoconvexity of $\left(u^{0}\right)^{t}\left(f(\cdot)+z^{t}(\cdot)\right)+\left(v^{0}\right)^{t} g_{A}+\left(w^{0}\right)^{t} h$, we have $\beta^{t}\left(\bar{x}-x^{0}\right)<0$ for any $\beta \in$ $\partial\left(\sum_{i \in M} u_{i}^{0}\left(f_{i}\left(x^{0}\right)+z_{i}^{t} x^{0}\right)+\sum_{j \in A} v_{j}^{0} g_{j}\left(x^{0}\right)+\sum_{k \in Q} w_{k}^{0} h_{k}\left(x^{0}\right)\right)$. We easily see that this contradicts $0 \in \sum_{i \in M} u_{i}^{0}\left(\partial f_{i}\left(x^{0}\right)+z_{i}\right)+\sum_{j \in A} v_{j}^{0} \partial g_{j}\left(x^{0}\right)+\sum_{k \in Q} w_{k}^{0} \partial h_{k}\left(x^{0}\right)$. Hence $x^{0}$ is a weak Paretooptimal solution of (MP).

Theorem 3.7. Let $\left(x^{0}, u^{0}, v^{0}, w^{0}\right)$ satisfy $\left(\mathrm{KKT}^{\prime}\right)$. If $\left(u^{0}\right)^{t}\left(f(\cdot)+z^{t}(\cdot)\right)+\left(v^{0}\right)^{t} g_{A}+\left(w^{0}\right)^{t} h$ is strictly pseudoconvex at $x^{0}$, and $f, g_{A}$, and h are regular at $x^{0}$, then $x^{0}$ is a Pareto-optimal solution of (MP).

The proof is similar to the one used for the previous theorem.

\section{Duality}

Following Mond and Weir [2], in this section we formulate a Wolfe-type dual problem (WD) and a Mond-Weir-type dual problem (MD) of the nonsmooth problem (MP) and establish duality theorems. We begin with a Wolfe-type dual problem:

$$
\begin{array}{ll}
\text { maximize } & f(y)+z^{t} y+v^{t} g(y) e+w^{t} h(y) e \\
\text { subject to } & 0 \in \sum_{i \in M} u_{i}\left(\partial f_{i}(y)+z_{i}\right)+\sum_{j \in P} v_{j} \partial g_{j}(y)+\sum_{k \in Q} w_{k} \partial h_{k}(y), \\
y \in \mathbb{R}^{n}, u \geq 0 \quad \text { with } u^{t} e=1, v \geqq 0, w \geqq 0 .
\end{array}
$$

Here $e=(1, \ldots, 1)^{t} \in \mathbb{R}^{m}$.

We now derive duality relations.

Theorem 4.1. Let $x$ be feasible for (MP) and $(y, u, v, w)$ feasible for (WD). If $f(\cdot)+z^{t}(\cdot)$, $g$ and $w^{t} h$ are convex, and $f$ is a regular function, then $f(x)+s(x \mid C) \nless f(y)+z^{t} y+v^{t} g(y) e+w^{t} h(y) e$.

Proof. Let $x$ be feasible for (MP) and $(y, u, v, w)$ feasible for (WD). Then $g(x) \leqq 0, h(x)=0$,

$$
0 \in \sum_{i \in M} u_{i}\left(\partial f_{i}(y)+z_{i}\right)+\sum_{j \in P} v_{j} \partial g_{j}(y)+\sum_{k \in Q} w_{k} \partial h_{k}(y)
$$


According to (4.1), there exist $\xi_{i} \in \partial f_{i}(y), \zeta_{j} \in \partial g_{j}(y)$, and $\alpha_{k} \in \partial h_{k}(y)$ such that

$$
\sum_{i \in M} u_{i}\left(\xi_{i}+z_{i}\right)+\sum_{j \in P} v_{j} \zeta_{j}+\sum_{k \in Q} w_{k} \alpha_{k}=0
$$

Assume that

$$
f(x)+s(x \mid C)<f(y)+z^{t} y+v^{t} g(y) e+w^{t} h(y) e .
$$

Multiplying (4.3) by $u$ and using the equality $z^{t} x=s(x \mid C)$, we have

$$
u^{t}\left(f(x)+z^{t} x\right)-u^{t}\left(f(y)+z^{t} y\right)-v^{t} g(y)-w^{t} h(y)<0
$$

since $u \geq 0$ and $u^{t} e=1$. Now by convexity of $f(\cdot)+z^{t}(\cdot), g$ and $w^{t} h$, we obtain

$$
\begin{gathered}
u^{t}\left(f(x)+z^{t} x\right)-u^{t}\left(f(y)+z^{t} y\right) \geqq \sum_{i \in M} u_{i}\left(\xi_{i}+z_{i}\right)^{t}(x-y), \quad \forall \xi_{i} \in \partial f_{i}(y), \\
v^{t} g(x)-v^{t} g(y) \geqq \sum_{j \in P} v_{j} \zeta_{j}^{t}(x-y), \quad \forall \zeta_{j} \in \partial g_{j}(y), \\
w^{t} h(x)-w^{t} h(y) \geqq \sum_{k \in Q} w_{k} \alpha_{k}^{t}(x-y), \quad \forall \alpha_{k} \in \partial h_{k}(y) .
\end{gathered}
$$

Since $v^{t} g(x) \leqq 0$ and $w^{t} h(x)=0$, we obtain the following inequality from (4.2), (4.5):

$$
u^{t}\left(f(x)+z^{t} x\right)-u^{t}\left(f(y)+z^{t} y\right)-v^{t} g(y)-w^{t} h(y) \geqq 0,
$$

which contradicts (4.4).

Hence the weak duality theorem holds.

Now we derive a strong duality theorem.

Theorem 4.2. Let $\bar{x}$ be a weak Pareto-optimal solution of (MP) at which a constraint qualification holds [14]. Then there exist $\bar{u} \in \mathbb{R}^{m}, \bar{v} \in \mathbb{R}^{p}$, and $\bar{w} \in \mathbb{R}^{q}$ such that $(\bar{x}, \bar{u}, \bar{v}, \bar{w})$ is feasible for (WD) and $z^{t} \bar{x}=s(\bar{x} \mid C)$. In addition, if $f(\cdot)+z^{t}(\cdot), g$ and $w^{t} h$ are convex, and $f$ is a regular function, then $(\bar{x}, \bar{u}, \bar{v}, \bar{w})$ is a weak Pareto-optimal solution of (WD) and the optimal values of (MP) and (WD) are equal.

Proof. From the (KKT) necessary optimality theorem [14], there exist $u \in \mathbb{R}^{m}, v \in \mathbb{R}^{p}$, and $w \in \mathbb{R}^{q}$ such that

$$
\begin{gathered}
0 \in \sum_{i \in M} u_{i}\left(\partial f_{i}(\bar{x})+z_{i}\right)+\sum_{j \in P} v_{j} \partial g_{j}(\bar{x})+\sum_{k \in Q} w_{k} \partial h_{k}(\bar{x}), \\
v^{t} g(\bar{x})=0, \quad u \geq 0, \quad v \geqq 0 .
\end{gathered}
$$


Since $u \geq 0$, we can scale the $u_{i}^{\prime} s, v_{j}^{\prime} s$ and $w_{k}^{\prime} s$ as follows:

$$
\bar{u}_{i}=\frac{u_{i}}{\sum_{i \in M} u_{i}}, \quad \bar{v}_{j}=\frac{v_{j}}{\sum_{i \in M} u_{i}}, \quad \bar{w}_{k}=\frac{w_{k}}{\sum_{i \in M} u_{i}} .
$$

Then $(\bar{x}, \bar{u}, \bar{v}, \bar{w})$ is feasible for (WD). Since $\bar{x}$ is feasible for (MP), it follows from Theorem 4.1 that

$$
\begin{gathered}
f(\bar{x})+s(\bar{x} \mid C)=f(\bar{x})+s(\bar{x} \mid C)+v^{t} g(\bar{x}) e+w^{t} h(\bar{x}) e \\
\nless f(y)+z^{t} y+v^{t} g(y) e+w^{t} h(y) e
\end{gathered}
$$

for any feasible solution $(x, u, v, w)$ of (WD). Hence $(\bar{x}, \bar{u}, \bar{v}, \bar{w})$ is a weak Pareto-optimal solution of (WD) and the optimal values of (MP) and (WD) are equal.

Remark 4.3. If we replace the convexity hypothesis of $f(\cdot)+z^{t}(\cdot)$ by strict convexity in Theorems 4.1 and 4.2, then these theorems hold for the case of a Pareto-optimal solution.

Remark 4.4. If we replace the convexity hypothesis of $w^{t} h$ by affineness of $h$ in Theorems 4.1 and 4.2 , then these theorems are also valid.

Theorem 4.5. Let $x$ be feasible for (MP) and $(y, u, v, w)$ feasible for (WD). If $u^{t}\left(f(\cdot)+z^{t}(\cdot)\right)+$ $v^{t} g+w^{t} h$ is pseudoconvex and $f, g$, and h are regular functions, then $f(x)+s(x \mid C) \not f(y)+z^{t} y+$ $v^{t} g(y) e+w^{t} h(y) e$.

Proof. Suppose to the contrary that $f(x)+s(x \mid C) \leq f(y)+z^{t} y+v^{t} g(y) e+w^{t} h(y) e$. By feasibility of $x$, we obtain

$$
u^{t}\left(f(x)+z^{t}(x)\right)+v^{t} g(x)+w^{t} h(x)<u^{t}\left(f(y)+z^{t}(y)\right)+v^{t} g(y)+w^{t} h(y) .
$$

Since $f, g$, and $h$ are regular functions, we have $\beta^{t}(x-y)<0$ by the pseudoconvexity of $u^{t}\left(f(\cdot)+z^{t}(\cdot)\right)+v^{t} g+w^{t} h$ for any $\beta \in \partial\left(\sum_{i \in M} u_{i}\left(f_{i}(y)+z_{i}^{t} y\right)+\sum_{j \in P} v_{j} g_{j}(y)+\sum_{k \in Q} w_{k} h_{k}(y)\right)$. This contradicts the feasibility of $(y, u, v, w)$. Hence the weak duality theorem holds.

Theorem 4.6. Let $\bar{x}$ be a weak Pareto-optimal solution of (MP) at which a constraint qualification holds [14]. Then there exist $\bar{u} \in \mathbb{R}^{m}, \bar{v} \in \mathbb{R}^{p}$, and $\bar{w} \in \mathbb{R}^{q}$ such that $(\bar{x}, \bar{u}, \bar{v}, \bar{w})$ is feasible for (WD) and $z^{t} \bar{x}=s(\bar{x} \mid C)$. If in addition $u^{t}\left(f(\cdot)+z^{t}(\cdot)\right)+v^{t} g+w^{t} h$ is pseudoconvex and $f, g$, and $h$ are regular functions, then $(\bar{x}, \bar{u}, \bar{v}, \bar{w})$ is a weak Pareto-optimal solution of (WD) and the optimal values of (MP) and (WD) are equal.

The proof is similar to the one used for Theorem 4.2.

Remark 4.7. If we replace the pseudoconvexity hypothesis of $u^{t}\left(f(\cdot)+z^{t}(\cdot)\right)+v^{t} g+w^{t} h$ by strictly pseudoconvexity in Theorems 4.5 and 4.6, then these results hold for the case of a Pareto-optimal solution. 
We now prove duality relations between (MP) and the following Mond-Weir-type dual problem:

$$
\begin{aligned}
\text { maximize } & f(y)+z^{t} y \\
\text { subject to } & 0 \in \sum_{i \in M} u_{i}\left(\partial f_{i}(y)+z_{i}\right)+\sum_{j \in P} v_{j} \partial g_{j}(y)+\sum_{k \in Q} w_{k} \partial h_{k}(y), \\
& v^{t} g(y)+w^{t} h(y) \geqq 0 \\
& y \in \mathbb{R}^{n}, u \geq 0 \quad \text { with } u^{t} e=1, v \geqq 0, w \geqq 0 .
\end{aligned}
$$

Theorem 4.8. Let $x$ be feasible for (MP) and $(y, u, v, w)$ feasible for (MD). If $f(\cdot)+z^{t}(\cdot)$, g and $w^{t} h$ are convex, and $f$ is a regular function, then $f(x)+s(x \mid C) \nless f(y)+z^{t} y$.

Proof. Let $x$ be feasible for (MP) and $(y, u, v, w)$ feasible for (MD). Then $g(x) \leqq 0, h(x)=0$,

$$
\begin{gathered}
0 \in \sum_{i \in M} u_{i}\left(\partial f_{i}(y)+z_{i}\right)+\sum_{j \in P} v_{j} \partial g_{j}(y)+\sum_{k \in Q} w_{k} \partial h_{k}(y), \\
v^{t} g(y)+w^{t} h(y) \geqq 0 .
\end{gathered}
$$

According to (4.11), there exist $\xi_{i} \in \partial f_{i}(y), \zeta_{j} \in \partial g_{j}(y)$, and $\alpha_{k} \in \partial h_{k}(y)$ such that

$$
\sum_{i \in M} u_{i}\left(\xi_{i}+z_{i}\right)+\sum_{j \in P} v_{j} \zeta_{j}+\sum_{k \in Q} w_{k} \alpha_{k}=0
$$

Assume that

$$
f(x)+s(x \mid C)<f(y)+z^{t} y
$$

Multiplying (4.14) by $u$ and using the inequality $z^{t} x \leqq s(x \mid C)$, we have

$$
u^{t}\left(f(x)+z^{t} x\right)<u^{t}\left(f(y)+z^{t} y\right)
$$

By convexity of $f(\cdot)+z^{t}(\cdot), g$ and $w^{t} h$, we obtain

$$
u^{t}\left(f(x)+z^{t} x\right)+v^{t} g(x)+w^{t} h(x) \geqq u^{t}\left(f(y)+z^{t} y\right)+v^{t} g(y)+w^{t} h(y) .
$$

From (4.12) and (4.16), we obtain

$$
u^{t}\left(f(x)+z^{t} x\right)-u^{t}\left(f(y)+z^{t} y\right) \geqq 0,
$$

since $v^{t} g(x) \leqq 0$ and $w^{t} h(x)=0$. However, (4.17) contradicts (4.15). Hence the proof is complete. 
Theorem 4.9. Let $\bar{x}$ be a weak Pareto-optimal solution of (MP) at which a constraint qualification holds. Then there exist $\bar{u} \in \mathbb{R}^{m}, \bar{v} \in \mathbb{R}^{p}$, and $\bar{w} \in \mathbb{R}^{q}$ such that $(\bar{x}, \bar{u}, \bar{v}, \bar{w})$ is feasible for (MD) and $z^{t} \bar{x}=s(\bar{x} \mid C)$. If in addition $f(\cdot)+z^{t}(\cdot), g$ and $w^{t} h$ are convex, and $f$ is a regular function, then $(\bar{x}, \bar{u}, \bar{v}, \bar{w})$ is a weak Pareto-optimal solution of (MD) and the optimal values of (MP) and (MD) are equal.

Proof. Let $\bar{x}$ be a weak Pareto-optimal solution of (MP) such that a constraint qualification is satisfied at $\bar{x}$. According to the (KKT) necessary optimality theorem, there exist $u \in \mathbb{R}^{m}$, $v \in \mathbb{R}^{p}$, and $w \in \mathbb{R}^{q}$ such that

$$
\begin{gathered}
0 \in \sum_{i \in M} u_{i}\left(\partial f_{i}(\bar{x})+z_{i}\right)+\sum_{j \in P} v_{j} \partial g_{j}(\bar{x})+\sum_{k \in Q} w_{k} \partial h_{k}(\bar{x}), \\
v^{t} g(\bar{x})=0, \quad u \geq 0, \quad v \geqq 0 .
\end{gathered}
$$

Since $u \geq 0$, we can scale the $u_{i}^{\prime} s, v_{j}^{\prime} s$, and $w_{k}^{\prime} s$ as in the proof of Theorem 4.2 such that $(\bar{x}, \bar{u}, \bar{v}, \bar{w})$ is feasible for (MD), it follows from Theorem 4.8 that $f(\bar{x})+s(\bar{x} \mid C) \nless f(y)+z^{t} y$ for any feasible solution $(y, u, v, w)$ of (MD). Hence $(\bar{x}, \bar{u}, \bar{v}, \bar{w})$ is a weak Pareto-optimal solution of (MD) and the optimal values of (MP) and (MD) are equal.

Remark 4.10. If we replace the convexity hypothesis of $f(\cdot)+z^{t}(\cdot)$ by strict convexity in Theorems 4.8 and 4.9, then these theorems hold in the sense of a Pareto-optimal solution.

Remark 4.11. If we replace the convexity hypothesis of $w^{t} h$ by affineness of $h$ in Theorems 4.8 and 4.9 , then these theorems are also valid.

Theorem 4.12. Let $x$ be feasible for (MP) and $(y, u, v, w)$ feasible for (MD). If $u^{t}\left(f(\cdot)+z^{t}(\cdot)\right)+$ $v^{t} g+w^{t} h$ is pseudoconvex and $f, g$, and h are regular functions, then $f(x)+s(x \mid C) \not f(y)+z^{t} y$.

Proof. Suppose that $f(x)+s(x \mid C) \leq f(y)+z^{t} y$. By using the feasibility assumptions and $z^{t} x \leqq s(x \mid C)$, we obtain

$$
u^{t}\left(f(x)+z^{t} x\right)+v^{t} g(x)+w^{t} h(x)<u^{t}\left(f(y)+z^{t} y\right)+v^{t} g(y)+w^{t} h(y) .
$$

By the same argument as in the proof of Theorem 4.5, we arrive at a contradiction.

Theorem 4.13. Let $\bar{x}$ be a weak Pareto-optimal solution of (MP) at which a constraint qualification holds. Then there exist $\bar{u} \in \mathbb{R}^{m}, \bar{v} \in \mathbb{R}^{p}$, and $\bar{w} \in \mathbb{R}^{q}$ such that $(\bar{x}, \bar{u}, \bar{v}, \bar{w})$ is feasible for (MD) and $z^{t} \bar{x}=s(\bar{x} \mid C)$. If in addition $u^{t}\left(f(\cdot)+z^{t}(\cdot)\right)+v^{t} g+w^{t} h$ is pseudoconvex and $f, g$, and $h$ are regular functions, then $(\bar{x}, \bar{u}, \bar{v}, \bar{w})$ is a weak Pareto-optimal solution of (MD) and the optimal values of (MP) and (MD) are equal.

The proof is similar to the one used for the previous theorem.

Remark 4.14. If we replace the pseudoconvexity hypothesis of $u^{t}\left(f(\cdot)+z^{t}(\cdot)\right)+v^{t} g+w^{t} h$ by strict pseudoconvexity in Theorems 4.12 and 4.13, then these results hold for the case of a Pareto-optimal solution. 


\section{Acknowledgment}

This research was supported by Basic Science Research Program through the National Research Foundation of Korea (NRF) funded by the Ministry of Education, Science and Technology (no. 2009-0074482).

\section{References}

[1] P. Wolfe, "A duality theorem for non-linear programming," Quarterly of Applied Mathematics, vol. 19, pp. 239-244, 1961.

[2] B. Mond and T. Weir, "Generalized concavity and duality," in Generalized Concavity in Optimization and Economics, S. Schaible and W. T. Ziemba, Eds., pp. 263-279, Academic Press, New York, NY, USA 1981.

[3] Q. H. Ansari, S. Schaible, and J.-C. Yao, " $\eta$-pseudolinearity," Rivista di Matematica per le Scienze Economiche e Sociali, vol. 22, no. 1-2, pp. 31-39, 1999.

[4] R. R. Egudo and M. A. Hanson, "On sufficiency of Kuhn-Tucker conditions in nonsmooth multiobjective programming," Technical Report M-888, Florida State University, Tallahassee, Fla, USA, 1993.

[5] V. Jeyakumar and B. Mond, "On generalised convex mathematical programming," Journal of the Australian Mathematical Society. Series B, vol. 34, no. 1, pp. 43-53, 1992.

[6] D. S. Kim and S. Schaible, "Optimality and duality for invex nonsmooth multiobjective programming problems," Optimization, vol. 53, no. 2, pp. 165-176, 2004.

[7] J. C. Liu, "Optimality and duality for generalized fractional programming involving nonsmooth pseudoinvex functions," Journal of Mathematical Analysis and Applications, vol. 202, no. 2, pp. 667-685, 1996.

[8] O. L. Mangasarian, Nonlinear Programming, McGraw-Hill, New York, NY, USA, 1969.

[9] S. K. Mishra and R. N. Mukherjee, "On generalised convex multi-objective nonsmooth programming," Australian Mathematical Society, vol. 38, no. 1, pp. 140-148, 1996.

[10] B. Mond and M. Schechter, "Nondifferentiable symmetric duality," Bulletin of the Australian Mathematical Society, vol. 53, no. 2, pp. 177-188, 1996.

[11] A. A. K. Majumdar, "Optimality conditions in differentiable multiobjective programming," Journal of Optimization Theory and Applications, vol. 92, no. 2, pp. 419-427, 1997.

[12] C. Singh, "Optimality conditions in multiobjective differentiable programming," Journal of Optimization Theory and Applications, vol. 53, no. 1, pp. 115-123, 1987.

[13] D. S. Kim, G. M. Lee, B. S. Lee, and S. J. Cho, "Counterexample and optimality conditions in differentiable multiobjective programming," Journal of Optimization Theory and Applications, vol. 109, no. 1, pp. 187-192, 2001.

[14] F. H. Clarke, Optimization and Nonsmooth Analysis, Canadian Mathematical Society Series of Monographs and Advanced Texts, John Wiley \& Sons, New York, NY, USA, 1983. 